\title{
Prospecting for Brown Dwarfs in Space Infrared Telescope Facility (SIRTF) Legacy Science Datasets
}

\author{
D. Padgett and J. O'Linger \\ SIRTF Science Center, California Institute of Technology, MC 220-6, \\ 1200 California Blvd., Pasadena, CA 91125 \\ K. Stapelfeldt \\ Jet Propulsion Laboratory, MC 183-900, 4800 Oak Grove Dr., \\ Pasadena, CA 91109
}

\begin{abstract}
We discuss the prospects for brown dwarf detections in the field and in star-forming regions using data from the SIRTF Legacy Science Programs. We concentrate in particular on the SIRTF Wide-area InfraRed Extragalactic survey (SWIRE) and "From Cores to Disks" program (c2d). The SWIRE program will map up to 70 square degrees of high galactic latitude sky, providing wavelength coverage from 3.6 to 160 $\mu \mathrm{m}$. SWIRE's estimated $5 \sigma$ sensitivity of 10 microJy at $4.5 \mu \mathrm{m}$ and its relatively large areal coverage will make it uniquely sensitive to the population of extremely cool $(\mathrm{T} \leq 600 \mathrm{~K})$ field brown dwarfs thus far undetected in other surveys. The "c2d" program will map up to 20 square degrees in nearby $(\mathrm{d} \leq 300 \mathrm{pc})$ star-forming molecular clouds over the wavelength range 3.6 - 70 microns, potentially detecting young brown dwarfs down to a few $\mathrm{M}_{\text {Jupiter }}$. Taken together, these two SIRTF datasets will offer a unique opportunity to take a census of the low mass/temperature members of the brown dwarf population.
\end{abstract}

\section{Introduction}

SIRTF (The Space InfraRed Telescope Facility) is an 85-cm cryogenic telescope which is currently scheduled for launch in early 2003. Diffraction limited at 6.5 $\mu \mathrm{m}$, SIRTF will provide imaging in 7 photometric bands from $3.6-160 \mu \mathrm{m}$ and spectroscopic capabilities from $5.3-94 \mu \mathrm{m}$. Utilizing a heliocentric orbit and warm launch with on-orbit telescope cooling, this mission hopes to achieve a 2.5 - 5 year mission lifetime before the liquid helium coolant is expended. With 10 - 1000 times the sensitivity of previous space infrared missions, SIRTF will be an ideal tool for the study of brown dwarfs.

\section{SIRTF Instrumentation}

SIRTF's short wavelength imaging instrument is the Infrared Array Camera (IRAC) designed by a team led by G. Fazio of the Smithsonian Astrophysical 
Observatory (SAO) and built at the Goddard Space Flight Center. IRAC provides wide-field $\left(5^{\prime} \mathrm{x} 5^{\prime}\right)$ images in four bands: $3.6 \mu \mathrm{m}, 4.5 \mu \mathrm{m}, 5.8 \mu \mathrm{m}$, and $8 \mu \mathrm{m}$. The instrument has two apertures, each of which images two bands simultaneously $(3.6 / 4.5 \mu \mathrm{m}$ and $5.8 / 8.0 \mu \mathrm{m})$. In order to cover all four bands, a single spacecraft pointing offset is required. The two shorter bands use $256 \mathrm{x}$ $256 \mathrm{InSb}$ detectors, while the longer bands use $256 \times 256 \mathrm{Si}$ :As arrays. The pixel size is $1.2^{\prime \prime}$, and the spatial resolution is limited by the telescope size (about $2^{\prime \prime}$ for $3.6 \mu \mathrm{m}$ ). IRAC is exquisitely sensitive; in a single 12 second exposure at low background, it should achieve $5 \sigma$ sensitivity of $27 \mu \mathrm{Jy}$ at $3.6 \mu \mathrm{m}, 34 \mu \mathrm{Jy}$ at 4.5 $\mu \mathrm{m}, 94 \mu \mathrm{Jy}$ at $5.8 \mu \mathrm{m}$, and $110 \mu \mathrm{Jy}$ at $8 \mu \mathrm{m}$.

SIRTF's long wavelength imaging instrument is the Multi-band Imaging Photometer for SIRTF (MIPS) designed and built by a team led by G. Rieke at the University of Arizona and Ball Aerospace. MIPS takes images at 24, 70, and $160 \mu \mathrm{m}$ and has the capability to obtain $\mathrm{R}=15$ spectra over the entire range from $55-96 \mu \mathrm{m}$. MIPS uses a $128 \times 128 \mathrm{Si}$ :As array with $2.55^{\prime \prime}$ pixels (PSF $\left.\mathrm{FWHM}=5.7^{\prime \prime}\right)$ for $24 \mu \mathrm{m}$ imaging of a $5^{\prime}$ by $5^{\prime} \mathrm{FOV}$. At $70 \mu \mathrm{m}$, MIPS uses a $32 \times 32 \mathrm{Ge}: \mathrm{Ga}$ detector with a choice of either $9.9^{\prime \prime}$ pixels (FOV $5^{\prime} \times 5^{\prime}$ ) or $4.99^{\prime \prime}$ pixels with $1 / 4$ the FOV area for superresolution of the $17^{\prime \prime}$ FWHM PSF. The SED mode uses the same array, with a slit size that covers $20^{\prime \prime} \times 4^{\prime}$ on the sky. Finally, MIPS can image at $160 \mu \mathrm{m}$ with a $2 \times 20$ stressed Ge:Ga array with $16^{\prime \prime}$ pixels and a $38^{\prime \prime}$ FWHM PSF. The array covers an area of $0.5^{\prime}$ by $5^{\prime}$ on the sky; however, the array is unfilled and requires multiple exposures to obtain a complete image. MIPS has a cryogenic scan mirror, which allows it to map large regions of the sky efficiently in all three bands via the freeze-frame scan mapping technique pioneered by 2MASS. The MIPS sensitivities depend heavily on the level and nature of the infrared background in the selected bands. However, at low background levels and a single 10 second exposure, MIPS hopes to achieve $5 \sigma$ sensitivities of $0.3 \mathrm{mJy}$ at $24 \mu \mathrm{m}, 3 \mathrm{mJy}$ at $70 \mu \mathrm{m}$, and $12.5 \mathrm{mJy}$ at $160 \mu \mathrm{m}$ (confusion limited). In the SED mode, MIPS should reach better than $100 \mathrm{mJy}$ $5 \sigma$ in a 10 second exposure.

SIRTF's primary spectroscopic instrument is the Infrared Spectrograph (IRS) designed by a team led by J. R. Houck of Cornell University, and built at Ball Aerospace. IRS has echelle spectrographs which cover the wavelength ranges from 10 - $19.5 \mu \mathrm{m}$ ("Short High" or SH) and $19-37 \mu \mathrm{m}$ ("Long High" or $\mathrm{LH}$ ) at $\mathrm{R}=600$. It also has $\mathrm{R}=50$ long-slit spectrographs which cover 5.3 - $14 \mu \mathrm{m}$ ("Short Low" or SL) and 14 - $40 \mu \mathrm{m}$ ("Long Low" or LL). The short wavelength arrays are $128 \times 128 \mathrm{Si}$ :As; the long wavelength channels use $128 \mathrm{x}$ $128 \mathrm{Si}: \mathrm{Sb}$ arrays. At low background levels, the expected mean $5 \sigma$ sensitivity in a 512 second integration is $0.5 \mathrm{mJy}$ for SL, $5 \mathrm{mJy}$ for $\mathrm{SH}, 2.5 \mathrm{mJy}$ for $\mathrm{LL}$, and $25 \mathrm{mJy}$ for LH. Updated parameters and sensitivities for all instruments, as well as instructions for proposers, can be found on the SIRTF Science Center Web site http://sirtf.caltech.edu.

\section{Brown Dwarf Science with SIRTF}

The "Design Reference Mission" used by the SIRTF project to determine science requirements included brown dwarfs as one of the four primary objectives for study. The choice of the two shortest bands for IRAC were partially dictated 
by the $3.4 \mu \mathrm{m} \mathrm{CH}_{4}$ band and $4.5 \mu \mathrm{m}$ flux peak expected in low temperature $\mathrm{T}$ dwarfs (Burrows et al. 1997). Recent studies suggest that $\mathrm{CO}$ absorption at $4.7 \mu \mathrm{m}$ will reduce the sensitivity of IRAC to brown dwarfs with $\mathrm{T} \geq 800 \mathrm{~K}$, but should not interfere with discovery of very low temperature brown dwarfs (Leggett et al. 2002). Using these two IRAC bands, it will be possible to quickly identify candidate brown dwarfs with $\mathrm{T}_{\text {eff }} \leq 1000 \mathrm{~K}$ by as objects which are faint or "drop out" at $3.6 \mu \mathrm{m}$, but are relatively bright at $4.5 \mu \mathrm{m}$. SIRTF's high sensitivity at the 8 and $24 \mu \mathrm{m}$ bands make it ideal for finding dust excesses around young brown dwarfs in nearby star formation regions. Indeed, a considerable amount of SIRTF guaranteed time is has been allocated for young brown dwarf searches and studies of known brown dwarfs.

\section{The SIRTF Legacy Program}

Seventy-five percent of SIRTF observing time will available for the general observer community. However, in the first two years of operation, 3160 hours of the observatory's time will be devoted to six "Legacy" projects pre-selected in 2000. The data collected by these large observing programs will be released immediately to the community through the SIRTF archive in order to enable rapid follow-on proposals. It is hoped that this strategy will help to optimize the use of SIRTF's limited lifetime in investigating new discoveries. Approximately twothirds of the Legacy time is devoted to extragalactic science, and the remainder is allocated for galactic investigations. Unfortunately, no Legacy Science project purely devoted to brown dwarf science was selected. Detailed information about SIRTF Legacy can be found on http://sirtf.caltech.edu/SSC/legacy/. The SIRTF Legacy projects are as follows:

GOODS: Great Observatories Origins Deep Survey; principal investigator (PI) Mark Dickinson (STScI); 647 hours. This project will observe distant galaxies with IRAC and the MIPS $24 \mu \mathrm{m}$ band. Although GOODS will go very deep with IRAC, the entire survey will cover only $300 \mathrm{arcmin}^{2}$, which is too small an area for a field brown dwarf search.

SINGS: SIRTF Nearby Galaxies Survey; PI Robert Kennicutt (U. Arizona); 512 hours. SINGS will perform imaging and spectral mapping of nearby large galaxies. Confusion from extragalactic extended structure makes this database unsuitable for field brown dwarf searches.

GLIMPSE: The SIRTF Galactic Plane Survey; PI Ed Churchwell (U. Wisconsin); 400 hours. Although GLIMPSE covers the largest area of any Legacy project $\left(200 \operatorname{deg}^{2}\right)$, its short IRAC exposures (4 seconds total) and potentially confused target regions within $\pm 1^{\circ}$ of the galactic plane decreases its utility for brown dwarf science.

FEPS: The Formation and Evolution of Planetary Systems; PI Michael Meyer (U. Arizona); 350 hours. FEPS is a targeted IRAC, MIPS, and IRS survey of young stars to determine the evolution of circumstellar material. Its use of sub-arcsecond IRAC exposures eliminates the possibility of serendipitous brown dwarf companion discoveries.

c2d: From Molecular Cores to Planet-forming Disks; PI Neal Evans (U. Texas); 400 hours. C2d is primarily devoted to using IRAC and MIPS to map 150 isolated cores and more than $20 \mathrm{deg}^{2}$ of nearby molecular clouds. Unfor- 
tunately, $160 \mu \mathrm{m}$ will be saturated in most fields. The regions to be mapped include clouds in Ophiuchus, Chamaeleon II, Serpens, Lupus, and Perseus. With 24 second IRAC exposures, c2d should achieve $30 \mu \mathrm{Jy}$ at $4.5 \mu \mathrm{m} 5 \sigma$ sensitivity, easily good enough to detect many young brown dwarf candidates in nearby clouds including isolated objects near $1 \mathrm{M}_{\text {Jupiter }}$ (Burrows et al. 1997). However, extended emission from PAHs $(8 \mu \mathrm{m})$ and dust $(24-70 \mu \mathrm{m})$ may compromise sensitivity to excesses around brown dwarf candidates. In addition, follow-up spectroscopy will be required to confirm faint $\mathrm{BD}$ candidates in this database, since at $1 \mathrm{Myr}$ age most $\mathrm{BD}$ are warm enough to mimic the spectra of late $\mathrm{M}$ stars.

SWIRE: SIRTF Wide-area Infrared Extragalactic Survey; PI Carol Lonsdale (IPAC/Caltech); 851 hours. SWIRE will map up to $70 \mathrm{deg}^{2}$ of low background sky with IRAC and MIPS. SWIRE will have the best Legacy database for serendipitous brown dwarf discoveries.

\section{Expected Brown Dwarf Detections from SWIRE}

SWIRE will have sufficient $4.5 \mu \mathrm{m}$ sensitivity and areal coverage to potentially detect very low mass old brown dwarfs in the field. The expected $5 \sigma$ sensitivities for SWIRE are $7.3 \mu \mathrm{Jy}$ at $3.6 \mu \mathrm{m}, 9.7 \mu \mathrm{Jy}$ at $4.5 \mu \mathrm{m}, 28 \mu \mathrm{Jy}$ at $5.8 \mu \mathrm{m}, 33 \mu \mathrm{Jy}$ at $8 \mu \mathrm{m}, 0.5 \mathrm{mJy}$ at $24 \mu \mathrm{m}, 3 \mathrm{mJy}$ at $70 \mu \mathrm{m}$, and $20 \mathrm{mJy}$ at $160 \mu \mathrm{m}$. Comparing these numbers to the predicted fluxes for old BD from Burrows et al. (1997), we find that SWIRE could detect a 5 Gyr old, $275 \mathrm{~K}$ brown dwarf at a distance of $10 \mathrm{pc}$ at $4.5 \mu \mathrm{m}$.

We simulated the detectability of field brown dwarfs in the SWIRE legacy survey using a Monte Carlo model similar to Martín et al. (2001), who estimated brown dwarf number counts for a range of IRAC survey sizes and sensitivities. Fluxes in the four IRAC bandpasses were tabulated vs. effective temperatures using the online models of Marley. $\mathrm{T}_{\text {eff }}$ vs. mass and age was taken from evolutionary models by A. Burrows. Brown dwarf initial mass functions with various power law indices $\alpha$ were simulated, with total counts normalized to the adopted stellar space density of $0.057 \mathrm{pc}^{-3}$ (Reid et al. 1999). An star formation rate proportional to $\left(1-\exp \left(-\mathrm{t} / \tau_{1}\right) \times \exp \left(-\mathrm{t} / \tau_{2}\right)\right.$ was used, with $\tau_{1}=0.2$ and $\tau_{2}=8.0$ Gyrs. Random masses, ages, and $\mathrm{X}, \mathrm{Y}, \mathrm{Z}$ coordinates within a suncentered cubical volume were then used as inputs to the evolution and mid-IR flux models, which were interpolated to give the observables. For $\alpha=1.5,1.0$, and 0.5 , the model predicts SWIRE detection of 23,8 , and 1 objects respectively in IRAC channel $2(4.5 \mu \mathrm{m})$.

\section{References}

Burrows, A., Marley, M., Hubbard, W.B. et al. 1997, ApJ, 491, 856

Leggett, S.K., Golimowski, D.A., Fan, X. et al. 2002, ApJ, 564, 452

Martín, E.L., Brandner, W., Jewitt, D.C. et al. 2001, PASP, 113, 529

Reid, I.N., Kirkpatrick, J.D., Liebert, J. et al. 1999, ApJ, 521, 613 